\title{
Flexible Assertive Community Treatment, Severity of Symptoms and Psy- chiatric Health Service Use, a Real life Observational Study
}

\author{
Marjan Drukker ${ }^{1, \dagger, *}$, Ellen Visser ${ }^{2, \dagger}$, Sjoerd Sytema ${ }^{2}$ and Jim van Os ${ }^{1,3}$
}

${ }^{1}$ Department of Psychiatry and Psychology, School for Mental Health and Neuroscience MHeNS, Maastricht University, p.o. box 616, Location Vijverdal, 6200 MD, Maastricht, the Netherlands

${ }^{2}$ Department of Psychiatry, University Medical Centre Groningen, University of Groningen, Rob Giel Centre, p.o. box 30.001, 9700 RB Groningen, the Netherlands

${ }^{3}$ King's College London, King's Health Partners Department of Psychosis Studies; Institute of Psychiatry, London, UK

\begin{abstract}
Background: Introduction of Flexible Assertive Community Treatment (FACT) may be associated with increased remission rates and changes in patterns of care. The present paper reports on differences in psychosocial functioning and health care use between patients in FACT and two groups of patients not currently provided with a specific model of community service.

Methods: The ongoing "Pharmacotherapy Monitoring and Outcome Survey" provided routine outcome measures of patients using antipsychotics in the north of the Netherlands. Level of psychosocial functioning was assessed using the Health of the Nations Outcome Scales (HoNOS) and matched with psychiatric health care consumption obtained from the Psychiatric Case Register. Patients who never received FACT, patients ever in FACT but not at assessment date, and patients in FACT were identified. Data were subjected to multilevel linear regression analysis.

Results: Data showed that most patients in FACT also had non-FACT episodes after the start of FACT. Furthermore, patients in FACT displayed higher levels of psychosocial functioning and used more outpatient care than the other two groups.

Conclusions: Patients in FACT receive more outpatient care and have better psychosocial functioning. However, causal inferences cannot be derived from these data. In addition, membership of a FACT-team in this setting did not last indefinitely.
\end{abstract}

Keywords: Assertive Community Treatment, Health Care, Population Register, Psychiatry, Psychosocial Factors, Severity of Illness.

\section{INTRODUCTION}

Flexible Assertive Community Treatment (FACT), a Dutch variant of Assertive Community Treatment (ACT), has been introduced throughout the Netherlands for patients with severe mental illness and other countries, such as the UK, are also adopting FACT [1]. The majority of severe mental illness patients $(75 \%)$ is diagnosed with schizophrenia or related psychotic disorder [2]. In ACT and FACT, teams coordinate a series of treatments according to the principles of assertive outreach. The aim of FACT is to decrease admissions and to prevent dropping out of care (no-care episodes) [3]. FACT thus should result in increased levels of outpatient care, in comparison with services not providing a particular model of community care, and previous work

\footnotetext{
*Address correspondence to this author at the Department of Psychiatry and Psychology, School for Mental Health and NeuroScience MHeNS, Maastricht University, p.o. box 616, location Vijverdal, 6200 MD Maastricht, the Netherlands; Tel: +31-(0)43-3883913; Fax: +31-(0)43-3884122

E-mail: Marjan.Drukker@MaastrichtUniversity.nl

${ }^{\dagger}$ Shared first authorship
}

focussing on this issue reported findings supporting this hypothesis [4]. In addition, FACT has been associated with increased symptomatic remission rates compared to care as usual $[5,6]$. If remission rates increase, severity of symptoms should decrease accordingly.

Whereas ACT only covers the $20 \%$ most severely ill group within the patients diagnosed with severe mental illness, FACT teams are delivering service for all patients with severe mental illness [3]. FACT combines two approaches within one multidisciplinary recovery-oriented team: (a) individual case management and home visits for 'extensive' care for patients who are currently stable and (b) shared caseload with 'intensive' full ACT approach for patients with more current needs. The care of the latter group includes daily reviews in staff meetings. The FACT teams are also in charge when patients receive care in the community and in hospitals. Teams decide whether patients can be discharged from or should be admitted to hospital [3]. FACT is a versatile and comprehensive care system with continuity of care as an important component. This is illustrated by the fact that when, with time, patients change in the level of need for care, they remain under the care of the same 
multidisciplinary team. In this way, FACT teams serve a diverse population of patients with severe mental illness and variable levels of need for care, whereas ACT teams only serve patients who are in crisis or have the highest needs for care. This leads to a lower frequency of contacts and a higher number of patients in FACT teams compared to ACT teams.

In the last ten years of the $20^{\text {th }}$ century, ACT teams were introduced in large Dutch cities. At the same time, FACT was introduced in some less urbanised areas such as the province of Groningen in the North of the country and in the province of Limburg in the South. Previous research on FACT was only performed in Limburg [4-6] and results need to be replicated in other areas. Within the Groningen area, not all patients diagnosed with severe mental illness in fact receive FACT; those not in FACT receive care as usual which constitutes the same treatment, however, without the benefit of coordination by a case-manager. Patients receiving care as usual are admitted when there is clinical exacerbation or relapse and they do not receive assertive outreach. Because in Groningen psychiatric health care consumption is registered and secondary data on routine outcome measures are available, data on mental health care consumption and level of psychosocial functioning could be combined while investigating differences between patients in FACT and patients not receiving care from FACT teams. Evaluation of psychosocial functioning was carried out at a random moment during ongoing treatment (i.e. not at baseline) and assessment of health care consumption was matched with this moment.

In order to examine FACT in circumstances of routine clinical practise, the following research questions were formulated:

1. Do subjects in FACT have better psychosocial functioning:

a. compared to patients receiving care as usual, who were never treated in FACT?

b. compared to patients receiving care as usual, who were ever in FACT?

2. Is mental health care consumption different when a patient is in FACT?

a. compared to patients receiving care as usual, who were never treated in FACT?

b. compared to patients receiving care as usual, who were ever in FACT?

\section{METHODOLOGY}

\section{Setting}

For the present analysis, data from the "Pharmacotherapy Monitoring and Outcome Survey" (PHAMOUS) were matched with mental health care consumption data. PHAMOUS is an ongoing survey initiated by the Rob Giel Research centre, including three large mental health care institutions and the University Centre of Psychiatry in Groningen with the three Northern provinces of The Netherlands as a catchment area. It combines a yearly somatic screening with routine outcome measures in patients diagnosed with schizophrenia or related psychotic disorder who are using antipsychotic medication. The Health of the Nation Outcome Scales (HoNOS) is part of this screening and is included in the treatment plans of these patients. The process of yearly monitoring all patients rather than a well-designed randomised controlled trial in a selected homogeneous subgroup within the total patient group can reflect real life patient outcomes and help evaluate and improve every day practice. The study was submitted for approval to the medical ethics committee of the University Medical Health Centre in Groningen. The committee's secretary stated that due to the nature of the study (i.e. being part of care as usual) formal approval was not required. PHAMOUS-participants who lived in the province of Groningen and who received care in the largest mental health care institution in the province of Groningen were included in this study. Patients could have up to three HoNOS assessments in the period of data collection used for the current analyses (January 2008 - June 2010).

\section{The HoNOS}

Nurses assessed the level of psychosocial functioning using the HoNOS [7]. This instrument includes 12 domains of functioning which are rated on a four item Likert scale between 0 (=no problem) to 4 (=(very) severe problem). A HoNOS total score as well as four subscores can be calculated (behaviour, impairment, symptoms, social) [8]. Good validity has been reported whereas reliability and sensitivity to change are deemed adequate [9].

\section{Psychiatric Case Register and the Matching Procedure}

Psychiatric Case Registers (PCR) register mental health care consumption, such as inpatient care, outpatient care and sheltered housing, separately for each individual among all mental health service users in a region. For the present analyses, data of the PCR registering mental health service consumption in the three Northern provinces of The Netherlands (population 1.7 million) was used. Since the PHAMOUS-study and the PCR both use the same anonymized patient codes, data of patients could be matched for use in this study. Ethical committees in Maastricht, Utrecht and Groningen have confirmed that by law routine outcome data collected for the purpose of management information is not within their remit as long as patients are aware of the purpose (including scientific publications).

Using PCR data between January 2000 and December 2009, each HoNOS assessment of each patient was defined as a FACT or a non-FACT assessment. To this end, FACTstatus at the HoNOS-assessment date was determined by defining FACT and non-FACT episodes. If a patient is in FACT treatment, he can be treated by one or more of several mental health care institutions in the region, but treatment is coordinated by the FACT case-manager and, therefore, all mental health care is provided within the framework of FACT. Patients should have a FACT contact at least once a month. If a patient missed two consecutive FACT contacts, it was assumed that services were no longer provided by the FACT team and, therefore, a FACT-episode ended if a subject had no FACT contact for $>61$ days. If patients were admitted for a period not exceeding 3 months directly after a FACT-episode, this admission was recoded to a FACTepisode, because short-term admissions are part of FACTtreatment. If a patient did not use any mental health care for 
100 days or more it was assumed that the patient moved or was outside the reach of FACT (e.g. forensic services). Therefore, periods in which no care was used were not included in the present analyses. Using the information from the episodes, FACT-status was determined for each HoNOS assessment: $0=$ control patients; never in FACT, $1=$ the patient was ever in FACT but not in a FACT-episode at assessment date, $2=$ the patient was in a FACT-episode at the date of assessment.

Proportion of inpatient days, outpatient contacts, day care/part-time care, sheltered housing and FACT contacts were calculated in the year after the PHAMOUS-assessment (i.e. the number of days a patient has contact with mental health care, e.g. inpatient day or outpatient contact, divided by total number of days in the period that is taken into account, e.g. 365). Proportion was chosen over number of days, because not all subjects were followed a full year. Assessments when patients were followed for less than 30 days (i.e. last date of health care consumption was shorter than 30 days after the last HoNOS assessment) were excluded from the analysis. This was the case when the HoNOS assessment was after October 2009 (PCR data being available until December 2009) or when patients did not receive care in the region.

\section{STATISTICAL ANALYSIS}

All analyses were performed using the statistical program Stata version 11 [10]. Because subjects could have up to three assessments which were all included in the analyses, the assumption of independence of the observations in standard regression techniques was not met. Multilevel linear regression analysis is ideally suited for the analysis of such structured data [11]. The regression coefficients obtained from this type of analysis can be interpreted in the same way as the estimates obtained from standard unilevel regression analyses. In both research questions, the main independent variable was the categorical FACT-group variable $(0=$ control, $1=$ patient ever in FACT, 2 patient currently in FACT). Age in categories (20-35, 36-45, 46-55, 56-76) and gender were included as confounders.

When assessing the association between FACT-status and level of psychosocial functioning, outcome variables were the HoNOS total scores and HoNOS subscores. When assessing the association between FACT-status and health care consumption, proportion of admissions, proportion of outpatient contacts, proportion of sheltered housing, proportion of day care/part-time care, and proportion of FACTcontacts in the year after the HoNOS-assessment were the dependent variable. Because mental health care consumption variables are highly skewed, a permute routine was performed to validate the results.

\section{RESULTS}

\section{Background characteristics}

Six-hundred forty-five patients were included; three of these were assessed three times, 130 twice, the remainder only once. Only 14 patients had HoNOS assessments when they were in FACT as well as HoNOS assessments when they were not in FACT. One-hundred and two (15.8\%) patients were controls, 97 ever-FACT patients had one or more assessments during a period they were not in FACT, and 460 had one or more assessments when they were in FACT. Only 20 non-FACT assessments in 19 ever-FACT patients were during a moment when the patient had not been in FACT before.

Sixty-six percent of the included patients were male, $23 \%$ was aged between 20 and 35 years and 20\% between 56 and 76 years; the remainder was aged between 36 and 55 years. The distribution of gender was similar in all three groups (Table 1, chi-square $=0.90, \mathrm{p}=0.64, \mathrm{df}=2$ ), but control patients were older (controls 2.9 years older than current FACT, $p=0.016$; no difference between ever-FACT and current-FACT: $B=-0.02 p=0.99)$. Mean HoNOS total score was 10.5 (standard deviation between $=5.5$, range $0-31$ ). In control patients, HoNOS total score was 12.5 , in ever-FACT patients 13.0 and in current FACT 9.5 (Table 1).

The duration of FACT episodes was on average 2.9 years (range 1 day - 10.0 years, Table 2), while HoNOS assessment was on average 3.0 years (range 1 day - 10.4 years) after the start of FACT. Only $10 \%$ of the assessments of patients in FACT were performed in the first 2 months of FACT-treatment (13\% in the first 3 months).

The frequency of a schizophrenia diagnosis and substance use was lower in controls compared to both the current FACT and the ever-FACT group, while a diagnosis in the category of affective and anxiety disorders was higher in controls (Table 1). There were no large or significant differences in duration of illness between the groups (Table 1; $\mathrm{F}=0.09, \mathrm{df}=2,593, \mathrm{p}=0.92$ ).

\section{FACT status and changes in FACT status over time}

Five hundred forty eight patients had FACT episodes and the mean number of FACT episodes was 3 (range 1-9; Table 2a). Patients who were in FACT at a certain moment in time (ever-FACT patients) also had non-FACT episodes after that moment. On average, ever-FACT patients had 3.7 nonFACT episodes (range 1-10) and most of these non-FACT episodes were after the first FACT episode.

\section{The Association Between Fact-Status and Health Care Use and Level of Psychosocial Functioning}

In the year after the HoNOS assessment, the proportion of time admitted was $10 \%$ in patients currently in FACT, while in control patients and the ever-FACT group this proportion was $48 \%$ and $50 \%$, respectively (Table $2 \mathbf{b}$ ). Patients currently in FACT had lower HoNOS total scores (better psychosocial functioning) and lower scores on the HoNOS subscales Symptoms and Social than control patients (Table 3). These current FACT-patients also had lower scores on HoNOS total and subscales than patients ever in FACT. Therefore, analyses of the association between health care use and FACT-status were controlled for level of functioning (Table 4). Patients currently in FACT were significantly fewer days admitted to hospital and had more outpatient, day care and FACT contacts. This was the case both for the comparison with control patients and the comparison with ever-FACT assessments, while there was no difference between the ever-FACT and the control assessments. 
Table 1. Sample Characteristics and HoNOS-Scores (Mean and Standard Deviation or Percentage)

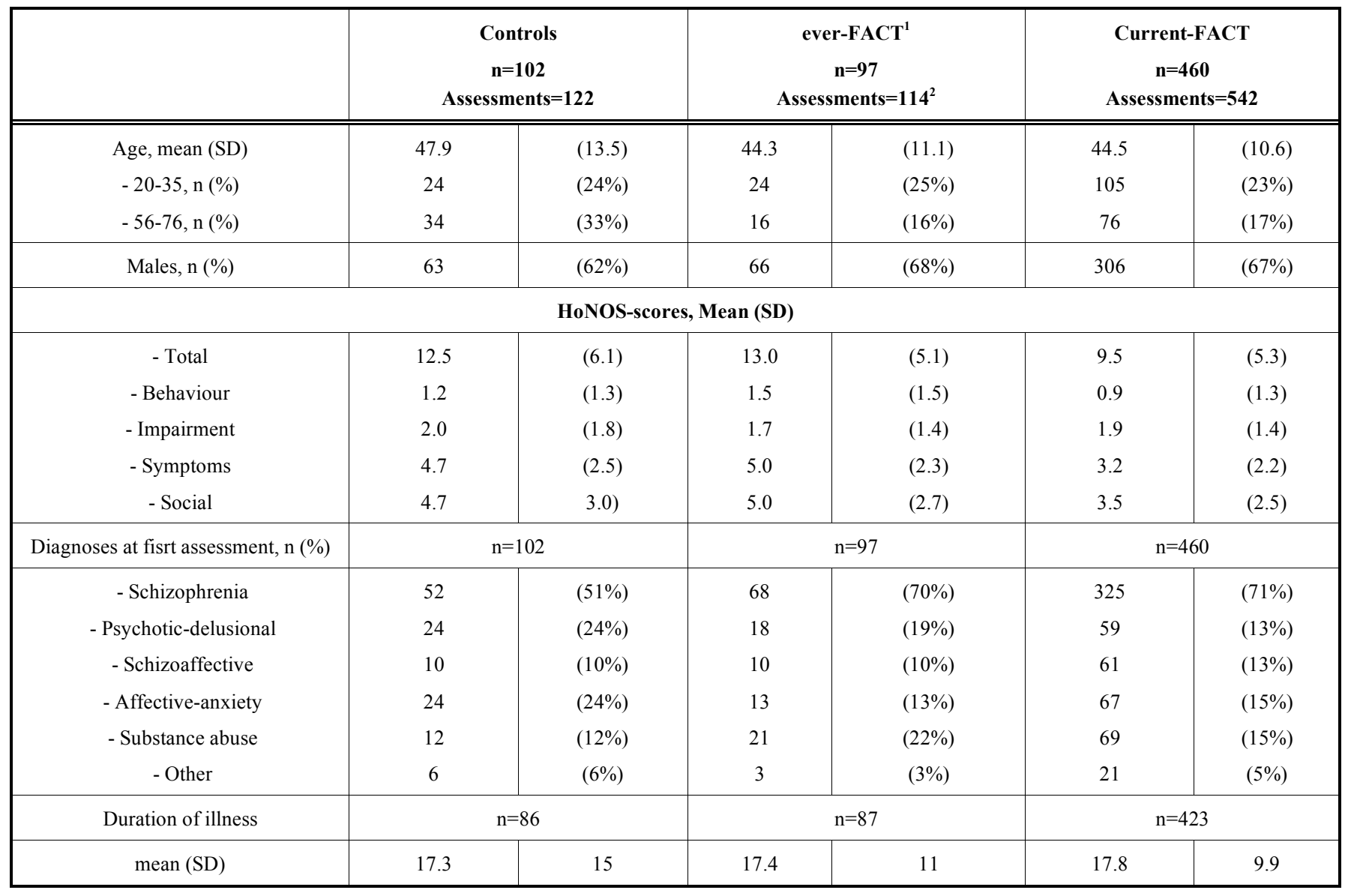

${ }^{1}$ Patients with at least one FACT-episode, but not in FACT at the moment of assessment

${ }^{2} 20$ assessments before FACT and 94 after FACT (or between two FACT periods).

Table 2a. Number of FACT and non-Fact Episodes, Total Lenght of Episde and Length of Episode at Assessment Date

\begin{tabular}{|c|c|c|c|c|c|c|c|c|c|}
\hline & \multicolumn{3}{|c|}{ Controls $n=102$ Episodes 168} & \multicolumn{3}{|c|}{$\begin{array}{c}\text { Ever-FACT }^{1} \text { n=505 } \\
\text { Episodes } 1336\end{array}$} & \multicolumn{3}{|c|}{$\begin{array}{c}\text { Current-FACT } \mathrm{n}=548 \\
\text { Episodes } 1092\end{array}$} \\
\hline & mean & range & $\mathbf{S D}^{2}$ & mean & range & SD & mean & range & SD \\
\hline $\begin{array}{c}\text { Number of episodes per patient } \\
\text { per treatment catagories }{ }^{3}\end{array}$ & 2.3 & $1-6$ & 1.5 & 3.7 & $1-10$ & 2.0 & 3.0 & $1-9$ & 1.4 \\
\hline \multirow[t]{2}{*}{$\begin{array}{l}\text { Length of episodes (All } \\
\text { episodes }^{3} \text { ) }\end{array}$} & 3.6 year & $\begin{array}{c}1 \text { day }-10.0 \\
\text { year }\end{array}$ & $4.2 \mathrm{y}$ & $\begin{array}{l}1.25 \\
\text { year }\end{array}$ & $\begin{array}{c}1 \text { day }-9.2 \\
\text { year }\end{array}$ & 1.9 year & 2.0 year & $\begin{array}{c}1 \text { day }-10.0 \\
\text { year }\end{array}$ & 2.7 year \\
\hline & \multicolumn{3}{|c|}{$\begin{array}{l}\text { Controls } n=75 \\
\text { Assessments } 75\end{array}$} & \multicolumn{3}{|c|}{$\begin{array}{l}\text { Ever-FACT } \mathrm{n}=80 \\
\text { Assessments } 83\end{array}$} & \multicolumn{3}{|c|}{$\begin{array}{c}\text { Current-FACT n=314 } \\
\text { Assessments } 322\end{array}$} \\
\hline \multirow[t]{2}{*}{$\begin{array}{c}\text { Length of episodes including a } \\
\text { HoNOS assessment }\end{array}$} & 5.7 year & $\begin{array}{c}1 \text { day }-10.0 \\
\text { year }\end{array}$ & 4.2 year & 2.6 year & $\begin{array}{c}1 \text { day }-8.8 \\
\text { year }\end{array}$ & 2.7 year & 2.9 year & $\begin{array}{c}1 \text { day }-10.0 \\
\text { year }\end{array}$ & 2.8 year \\
\hline & & & & & & & \multicolumn{3}{|c|}{$\begin{array}{c}\text { Current-FACT n=464 } \\
\text { Assessments } 642\end{array}$} \\
\hline $\begin{array}{l}\text { Length of FACT episodes At the } \\
\text { moment of HoNOS assessment }\end{array}$ & & & & & & & 3.0 year & $\begin{array}{c}1 \text { day }-10.4 \\
\text { year }\end{array}$ & 3.0 year \\
\hline
\end{tabular}

${ }^{1}$ Patients with at least one episode of FACT, but not in FACT at the moment of assessment.

${ }^{2}$ Standard deviation between (multilevel data)

${ }^{3}$ In total, 2596 episodes in 650 patients

${ }^{4}$ In total, 480 assessments in 460 patients

${ }^{5}$ In total, 884 assessments in 650 patients; 2010 HoNOS assessments included as if last episode continued. 
Table 2b. Health Care Consumption in the Year after HoNOS Assessment by FACT Status at HoNOS Assessment

\begin{tabular}{|c|c|c|c|c|c|c|c|c|c|}
\hline \multirow[t]{2}{*}{ Health care consumption ${ }^{1}$ : } & \multicolumn{3}{|c|}{$\begin{array}{l}\text { Controls } n=70 \\
\text { Assessments } 70\end{array}$} & \multicolumn{3}{|c|}{$\begin{array}{c}\text { Ever-FACT }^{2} \text { n=77 } \\
\text { Assessments } 80\end{array}$} & \multicolumn{3}{|c|}{$\begin{array}{c}\text { Current-FACT } n=280 \\
\text { Assessments } 304\end{array}$} \\
\hline & mean & median & $\mathrm{SD}^{3}$ & mean & median & SD & mean & median & SD \\
\hline$\%$ Admitted & 48.3 & 39.7 & 40.0 & 50.0 & 48.3 & 41.0 & 10.4 & 0 & 28.0 \\
\hline$\%$ Outpatient contacts & 1.6 & 0 & 3.6 & 3.5 & 1.3 & 4.3 & 7.9 & 6.4 & 6.0 \\
\hline$\%$ Sheltered housing & 0 & 0 & 0 & 1.8 & 0 & 12.6 & 4.6 & 0 & 20.9 \\
\hline$\%$ Day care / part time & 15.2 & 0 & 29.3 & 13.5 & 0 & 26.1 & 29.7 & 0 & 41.2 \\
\hline$\%$ FACT-contacts & 0 & 0 & 0 & 3.6 & 0.3 & 10.4 & 27.3 & 11.2 & 33.3 \\
\hline
\end{tabular}

${ }^{1}$ In total, 454 assessments in 419 patients

${ }^{2}$ Patients with at least one episode of FACT, but not in FACT at the moment of assessment

${ }^{3}$ Standard deviation between (multilevel data)

Table 3. Association (Regression Coefficients) Between FACT and Level of Psychosocial Functioning (HoNOS) ${ }^{1}$ Controlled for Age (Categories) and Gender (745 Assessments in 622 Patients) ${ }^{2}$

\begin{tabular}{|c|c|c|c|c|c|c|c|c|c|c|}
\hline & \multicolumn{2}{|c|}{ HoNOS total } & \multicolumn{2}{|c|}{ Behaviour } & \multicolumn{2}{|c|}{ Impairment } & \multicolumn{2}{|c|}{ Symptoms } & \multicolumn{2}{|c|}{ Social } \\
\hline & B & $95 \% \mathrm{CI}$ & B & $95 \% \mathrm{CI}$ & B & $95 \% \mathrm{CI}$ & $\mathbf{B}$ & $95 \% \mathrm{CI}$ & $\mathbf{B}$ & $95 \%$ CI \\
\hline Controls & 0.0 & ref & 0.0 & ref & 0.0 & ref & 0.0 & ref & 0.0 & ref \\
\hline Ever-FACT & 0.57 & $-0.94 ; 2.1$ & 0.25 & $-0.12 ; 0.63$ & -0.15 & $-0.55 ; 0.25$ & 0.21 & $-0.41 ; 0.84$ & 0.34 & $-0.40 ; 1.07$ \\
\hline Current-FACT & $-2.79^{* * *}$ & $-3.98 ;-1.6$ & -0.14 & $-0.43 ; 0.16$ & -0.04 & $-0.35 ; 0.28$ & $-1.44^{* * *}$ & $-1.93 ;-0.95$ & $-1.14^{* * *}$ & $-1.72 ;-0.57$ \\
\hline Current vs. Ever & \multicolumn{2}{|c|}{$\chi^{2}=32.6 \mathrm{df}=1 \mathrm{p}<0.001$} & \multicolumn{2}{|c|}{$\chi^{2}=7.1 \mathrm{df}=1 \mathrm{p}=0.0079$} & \multicolumn{2}{|c|}{$\chi^{2}=0.55 \mathrm{df}=1 \mathrm{n} . \mathrm{s}}$. & \multicolumn{2}{|c|}{$\chi^{2}=46.2 \mathrm{df}=1 \mathrm{p}<0.001$} & \multicolumn{2}{|c|}{$\chi^{2}=26.9 \mathrm{df}=1 \mathrm{p}<0.001$} \\
\hline
\end{tabular}

${ }^{1}$ A higher HoNOS-score represents a lower level of psychosocial functioning

293 controls (111 assessments); 90 ever-FACT (103 assessments); 423 current FACT (491 assessments)

${ }^{* * *} \mathrm{p}<.001$

ref: reference category

Table 4. Association Between FACT and Health Care Consumption, Controlled for Level of Psychosocial Functioning (Regression Coefficients)

Psychosocial Functioning Assessed with the HoNOS Total Score; 404 Assessments in 376 Patients $^{1}$

\begin{tabular}{|c|c|c|c|c|c|c|c|c|c|c|}
\hline & \multicolumn{2}{|c|}{$\%$ Admitted } & \multicolumn{2}{|c|}{$\%$ Outpatient Contacts } & \multicolumn{2}{|c|}{ \% Sheltered Housing } & \multicolumn{2}{|c|}{$\%$ Day care / part-time } & \multicolumn{2}{|c|}{$\%$ FACT-contacts } \\
\hline & $\mathbf{B}$ & $95 \% \mathrm{CI}$ & $\mathbf{B}$ & $95 \%$ CI & $\mathbf{B}$ & $95 \%$ CI & $\mathbf{B}$ & $95 \%$ CI & $\mathbf{B}$ & $95 \%$ CI \\
\hline Controls & 0 & ref & 0 & Ref & 0 & ref & 0 & ref & 0 & ref \\
\hline Ever-FACT $^{1}$ & 2.7 & $-8.2 ; 13.5$ & 1.7 & $-0.2 ; 3.6$ & 2.1 & $-4.2 ; 8.4$ & 0.9 & $-12.1 ; 13.9$ & 8.3 & $-1.3 ; 18.0$ \\
\hline Current-FACT & -34.6 & $-43.6 ;-25.6^{* * *}$ & 6.5 & $5.0 ; 8.1^{* * *}$ & 4.8 & $-0.6 ; 10.1$ & 14.0 & $3.0 ; 25.0^{*}$ & 29.1 & $21.0 ; 37.2^{* * *}$ \\
\hline Overall $\chi^{2}(\mathrm{df}=2)$ & \multicolumn{2}{|c|}{$\chi^{2}=104.1 \mathrm{p}<0.001$} & \multicolumn{2}{|c|}{$\chi^{2}=92.5 \mathrm{p}<0.001$} & \multicolumn{2}{|c|}{$\chi^{2}=3.65 \mathrm{p}=0.16$} & \multicolumn{2}{|c|}{$\chi^{2}=10.8 \mathrm{p}=0.004$} & \multicolumn{2}{|c|}{$\chi^{2}=69.6 \mathrm{p}<0.001$} \\
\hline Permute & \multicolumn{2}{|c|}{$\chi 2=104.1, \mathrm{p}<0.001$} & \multicolumn{2}{|c|}{$\chi 2=92.5, p<0.001$} & \multicolumn{2}{|c|}{$\chi 2=3.65, p=0.13$} & \multicolumn{2}{|c|}{$\chi 2=10.8, p=0.007$} & \multicolumn{2}{|c|}{$\chi 2=69.6, p<0.001$} \\
\hline $\begin{array}{l}\text { Current vs. Ever } \\
\qquad(\mathrm{df}=1)\end{array}$ & \multicolumn{2}{|c|}{$\chi^{2}=72.1 \mathrm{p}<0.0001$} & \multicolumn{2}{|c|}{$\chi^{2}=45.2 \mathrm{p}<0.0001$} & \multicolumn{2}{|c|}{$\chi^{2}=1.29 \mathrm{p}=0.26$} & \multicolumn{2}{|c|}{$\chi^{2}=6.9 \mathrm{p}=0.008$} & \multicolumn{2}{|c|}{$\chi^{2}=33.8 \mathrm{p}<0.001$} \\
\hline Permute & \multicolumn{2}{|c|}{$\chi^{2}=72.1, \mathrm{p}<0.001$} & \multicolumn{2}{|c|}{$\chi^{2}=45.2, p<0.001$} & \multicolumn{2}{|c|}{$\chi^{2}=1.29, \mathrm{p}=0.25$} & \multicolumn{2}{|c|}{$\chi^{2}=6.9, p=0.009$} & \multicolumn{2}{|c|}{$\chi 2=33.8, p<0.001$} \\
\hline
\end{tabular}

B: Regression coefficients, proportion of days per year

${ }^{1} 62$ controls (62 assessments), 68 ever FACT (69 assessments), 252 FACT (273 assessments)

${ }^{2}$ Patients with at least one FACT-episode, but not in FACT at the moment of assessment

${ }^{*} \mathrm{p}<0.05$

${ }^{* *} \mathrm{p}<.01$

p*** $<.001$

ref: reference category 


\section{DISCUSSION}

Patients in FACT received less inpatient and more outpatient care, even when level of functioning was taken into account. In addition, FACT patients had higher levels of psychosocial functioning than ever-FACT patients and control patients, in particular in the domains Symptoms and Social, but it is impossible to make causal inferences. Therefore, the most important finding of the present analysis may be that despite the principle of continuity of care, FACT episodes and non-FACT episodes alternate in a substantial part of the FACT caseload. Short-term admissions are not an explanation for this finding, because in the data short term admissions were included in the previous FACT episode.

\section{Methodological issues}

The present study used secondary data matched with PCR data. The HoNOS was assessed as part of a yearly screening of patients who use antipsychotic medication. In this real life data set, duration of FACT varied between 1 day and 10 years. Average duration of FACT at the time of the HoNOS assessment was 3.0 years and only $10 \%$ of the HoNOS assessments were performed within 2 months after the start of FACT. However, at the time of the PHAMOUSstudy, FACT was already in place and, therefore, data only included 20 baseline assessments, precluding a pre-post comparison.

Although the study population consisted of severe mental illness patients using antipsychotics, and duration of illness was similar across the three groups, groups may have been differed from each other at baseline, which was not examined. In the present data collection, patients living in the vicinity of a FACT team were more likely to be treated in FACT. However, refusal and long-term admission may also constitute valid reasons why patients were in the control group or in the ever-FACT group (at the moment of the HoNOS assessment). Furthermore, analysis of the data showed differences in diagnostic distribution between current-FACT and ever-FACT on the one hand and controls on the other; it is likely that such differences already existed at baseline. Thus, although results show that FACT patients have better outcomes, we cannot make causal inferences. However, differences in diagnostic distribution between the current FACT and the ever FACT group were small, while in the analyses, current FACT patients were compared with both ever-FACT patients and controls, and results were rather similar.

Although 127 patients were assessed twice, only 52 of these longitudinal assessments are in one episode or in two adjacent episodes of which the second one is a FACTepisode. Although the power is relatively low, a post hoc longitudinal analysis showed that impairment scores deteriorated in the controls $(B=1.2 p=0.02, n=5)$ but not in the patients in a FACT-episode $(\mathrm{B}=-0.18, \mathrm{p}=0.36, \mathrm{n}=42$; everFACT assessments: $\mathrm{B}=0.3, \mathrm{p}=0.95, \mathrm{n}=5$; overall chi-square of interaction term $=6.2, \mathrm{df}=4, \mathrm{p}=0.045)$. Because the difference between FACT and ever-FACT was smaller and because the numbers in this post hoc analysis were low, these results are hypothesis generating at best.
In addition, the present analysis has several limitations. First, in these secondary data, FACT as a whole was evaluated. We did not differentiate between the $20 \%$ most severely ill patients, who are treated by the principles of ACT and the other patients in FACT, with less intensive treatment. Second, control patients were older than current FACT-patients and this is in agreement with other FACT results [5]. Possibly, patients were more likely to be selected for FACT-treatment when they were younger. Fortunately, there was no difference in age between current-FACT and ever-FACT, while results of current FACT compared with control groups were similar. In addition, all analyses were controlled for age group.

Third, data did not include at least one FACT and one non-FACT assessment for each patient ever in FACT. The large majority of FACT-patients only had HoNOSassessments during FACT-episodes, while other FACT patients only had assessments after FACT. These limitations result from the use of secondary data. However, these secondary data combined with mental health care use collected by the PCR provide results that resemble a study of "real-life" routine clinical practise as opposed to a Randomised Controlled Trail (RCT). The PHAMOUS-study included all patients using antipsychotics, rather than a subsample with strict inclusion and exclusion criteria, as typically selected in the context of an RCT.

Because data on mental health care use are skewed, the assumption of normal distribution of the dependent variable, needed for linear regression, was not met. Therefore, a sensitivity analysis was performed using the Stata command PERMUTE. All models analysing the outcome of mental health care use were performed 10000 times, creating a distribution of chi-square values. All p-values were similar to the original analyses.

Finally, FACT-status was obtained from the PCR and, therefore, not available for 2010. Therefore, for assessments between January and June 2010, FACT status of December $31^{\text {st }} 2009$ was extrapolated. Thus, patients that did change treatment in 2010 introduced some noise.

\section{Other FACT Research}

FACT is a Dutch variant of ACT [3] and although it has been implemented across the country, research results to date mainly originated from one province in the South of the Netherlands, Limburg. Using Limburg data, it has been shown that remission rates are larger in FACT than in care without a specific community component [5] when there is a need for care with respect to psychotic symptoms. The present data originate from another Dutch area and also show better functioning of patients in FACT, but this analysis cannot be seen as a replication, because baseline assessment is missing (see above). On the other hand, because of the continuity-of-care principle, costs were higher in FACT in Limburg; the decrease in costs of inpatient care was outweighed by the costs of patients remaining in outpatient care [4]. The present results also show lower levels of inpatient care and higher levels of outpatient care in FACT. Thus, in the short term, outpatient care as well as the total psychiatric health care costs increase because of the continuity-of-care principle. However, this principle aims to save health care costs in 
the longer term, by reduction of symptoms levels and number of admissions. The longitudinal data used in the present paper and in Limburg do not span the time period needed to evaluate these long-term effects. If these data are available in future, researchers should not evaluate the overall or average changes in health care use over e.g. 10 years, but they should categorise the period to evaluate short-term and long-term health care use.

Results of ACT are also promising both in the Netherlands and in other countries [12-16]. Previously, the choice between FACT and ACT was merely based on characteristics of the region. FACT-teams were introduced in regions where the number of patients in the most severely ill subgroup was not sufficient to start an ACT-team (e.g. rural areas). Recently, the first study that compared effectiveness of FACT with effectiveness of ACT was published [1]. Various outcomes with respect to admission days showed a reduction after the transformation from Assertive Outreach to community mental health teams adopting the principles of FACT; staff suggested that patients that were unnecessarily retained in assertive outreach could be better served in FACT [1]. Unfortunately, this study did not include psychopathology or other relevant patient outcomes.

\section{Once in FACT always in FACT?}

FACT aims to keep patients in care [3] and in actual practice FACT experts generally assume that patients admitted to FACT, remain in FACT. If this were the case, the data should have shown a single prolonged episode of FACT care for each patient. In actual fact, however, patients on average had 3 separate FACT episodes, which alternated with periods of non-FACT episodes or no treatment. FACT care in practice thus is more flexible than generally assumed, at least in our data. This finding has been shown in ACT-research as well [17-19]. Although FACT explicitly aims to keep patients in the same care setting despite fluctuations in severity, membership of a FACT-team does not last indefinitely. One explanation may be that patients sometimes are admitted to sheltered housing facilities within a psychiatric hospital, where treatment logically is not combined with FACT. Another reason may be that some patients deteriorate and are admitted for a long period (admissions not exceeding 3 months were still within the remit of FACT in the analyses). Patients may also move outside the area of the FACT-team or patients may leave FACT because they improve sufficiently to migrate to a less intensive form of care. However, the frequency of the HoNOS-assessments is too low to assess the proportion of patients who drop out of FACT because of an improvement in functioning. Previous research showed that $24 \%$ of patients discharged from ACT were transferred to less intensive care and $17 \%$ to more intensive care [19]. In theory, the proportion referred to less intensive care should be lower in FACT, because FACT also caters for patients requiring less intensive care.

\section{CONCLUSION}

Patients in FACT, included in the present study, received less inpatient and more outpatient care than ever-FACT patients and control patients. In addition, FACT patients had higher levels of psychosocial functioning than ever-FACT patients and control patients, in particular in the domains
Symptoms and Social. However, the structure of the present data precludes causal inference. Furthermore, we may conclude that membership of a FACT-team does not last indefinitely.

\section{TRIAL REGISTRATION}

The study was submitted for approval to the medical ethics committee of the University Medical Health Centre in Groningen. The committee's secretary stated that due to the nature of the study (i.e. being part of care as usual) formal approval was not required.
ABBREVIATIONS
ACT $=$ Assertive Community Treatment,
FACT $=$ Flexible Assertive Community Treatment,
HoNOS $=$ Health of the Nations Outcome Scales,
PHAMOUS $=$ Pharmacotherapy Monitoring and Out- come Survey,
PCR = Psychiatric Case Register,
RCT $=$ Randomized controlled trial,
SD $=$ standard deviation

\section{CONFLICT OF INTEREST}

The authors confirm that this article content has no conflicts of interest.

\section{ACKNOWLEDGEMENTS}

We gratefully acknowledge the financial support of the PCR by the ministry of Health, Welfare and Sport.

\section{REFERENCES}

[1] Firn M, Hindhaugh K, Hubbeling D, Davies G, Jones B, White SJ. A dismantling study of assertive outreach services: comparing activity and outcomes following replacement with the FACT model. Soc Psychiatr Epidemiol 2012. Available from http://link.springer.com/article/10.1007\%2Fs00127-012-0602-x

[2] Drukker M, Bak MLFJ, Campo JA, Driessen G, Van Os J, Delespaul PAEG. The Cumulative Needs for Care Monitor (CNCM), a unique monitoring system in the South of The Netherlands. Soc Psychiatr Epidemiol 2010; 45(4): 475-85.

[3] Van Veldhuizen JR. FACT: a Dutch version of ACT. Commun Ment Health J 2007; 43(4): 421-33.

[4] Drukker M, Van Os J, Sytema S, Driessen G, Visser E, Delespaul P. Function Assertive Community Treatment (FACT) and psychiatric service use in patients diagnosed with severe mental illness. Epidemiol Psychiatr Sci 2011; 20(3): 273-8.

[5] Drukker M, Maarschalkerweerd M, Bak MLFJ, et al. A real-life observational study to the effectiveness of F-ACT in a Dutch mental health region. BMC Psychiatr 2008; 8: 93.

[6] Bak M, Van Os J, Delespaul PAEG, et al. An observational, "real life" trial of the introduction of assertive community treatment in a geographically defined area using clinical rather than service use outcome criteria. Soc Psychiatr Epidemiol 2007; 42(2): 125-30.

[7] Phamous. Phamous pharmacotherapy monitoring and outcome survey. Available at. [http://www.phamous.eu/meerover.html] Groningen; 2013 [retreived 2013 January 22];

[8] Wing J, Curtis RH, Beevor A. Health of the Nation Outcome Scales (HoNOS). Glossary for HoNOS score sheet. Br J Psychiatr 1999; 174: 432-4.

[9] Pirkis JE, Burgess PM, Kirk PK, Dodson S, Coombs TJ, Williamson MK. A review of the psychometric properties of the 
Health of the Nation Outcome Scales (HoNOS) family of measures. Health Qual Life Outcomes 2005; 3: 76.

[10] Statacorp. Stata Statistical Software (Version 11). College Station, Texas: Stata Corporation 2009.

[11] Snijders T, Bosker R. Multilevel analysis, an introduction to basic and advanced modeling. London: SAGE Publications 1999.

[12] Marshall M, Lockwood A. Assertive community treatment for people with severe mental disorders. Cochrane Database Syst Rev 2000; (2): CD001089.

[13] Sytema S, Wunderink L, Bloemers W, Roorda L, Wiersma D. Assertive community treatment in the Netherlands; a randomized controlled trial. Acta Psychiatr Scand 2007; 116(2): 105-12.

[14] Ito J, Oshima I, Nishio $\mathrm{M}$, et al. The effect of Assertive Community Treatment in Japan. Acta Psychiatr Scand 2011; 123(5): 398-401.

[15] Bhugra D, Ayonrinde O, Butler G, Leese M, Thornicroft G. A randomised controlled trial of assertive outreach vs. treatment as usual for black people with severe mental illness. Epidemiol Psychiatr Sci 2011; 20(1): 83-9.

[16] Nordén T, Malm U, Norlander T. Resource Group Assertive Community Treatment (RACT) as a Tool of Empowerment for Clients with Severe Mental Illness: A Meta-Analysis. Clin Pract Epidemiol Ment Health 2012; 8: 144-51.

[17] Kortrijk HE, Mulder C, Drukker M, Wiersma D, Duivenvoorden H. Duration of assertive community treatment and the interpretation of routine outcome data. Aust N Z J Psychiatr 2012; 46(3): 240-8.

[18] Kortrijk HE, Mulder CL, Roosenschoon BJ, Wiersma D. Treatment outcome in patients receiving assertive community treatment. Commun Ment Health J 2010; 46(4): 330-6.

[19] Mulder CL, Kortrijk HE. [Effects of treatment duration on the interpretation of ROM data in ACT patients] De invloed van de duur van behandeling op het interpreteren van ROM-metingen bij ACT. Tijdschr Psychiatr 2012; 54(2): 191-6.

Received: May 15, 2013

(C) Drukker et al.; Licensee Bentham Open.

This is an open access article licensed under the terms of the Creative Commons Attribution Non-Commercial License (http://creativecommons.org/licenses/by-nc/3.0/) which permits unrestricted, non-commercial use, distribution and reproduction in any medium, provided the work is properly cited. 